\title{
Novel regenerative therapy using cell-sheet covered with omentum flap delivers a huge number of cells in a porcine myocardial infarction model
}

\author{
Yasuhiro Shudo, MD, ${ }^{a}$ Shigeru Miyagawa, MD, PhD, ${ }^{a}$ Satsuki Fukushima, MD, PhD, ${ }^{\mathrm{a}}$ \\ Atsuhiro Saito, $\mathrm{PhD},{ }^{\mathrm{a}}$ Tatsuya Shimizu, MD, PhD, ${ }^{\mathrm{b}}$ Teruo Okano, $\mathrm{PhD},{ }^{\mathrm{b}}$ and Yoshiki Sawa, MD, $\mathrm{PhD}^{\mathrm{a}}$
}

\begin{abstract}
Objective: A key challenge to applying cell transplantation to treat severely damaged myocardium is in delivering large numbers of cells with minimum cell loss. We developed a new implantation method using skeletal myoblast (SMB) sheets, wrapped with an omentum flap as a blood supply to deliver huge numbers of SMBs to the damaged heart. We examined whether this method could be used to deliver a large amount of cells to deteriorated porcine myocardium.
\end{abstract}

\begin{abstract}
Methods: Cell sheets were obtained by culturing mini-pig autologous SMB cells on temperature-responsive culture dishes. Myocardial infarction was induced by placing an ameroid constrictor around the left anterior descending artery. The mini-pigs were divided into 4 treatment groups $(n=6$ in each): cell sheets with omentum, cell sheets only, omentum only, and sham operation. Each animal implant consisted of 30 cell sheets $\left(1.5 \times 10^{7}\right.$ cells per sheet). Six 5-layer constructs were each placed on a different area, immediately adjacent to but not overlapping one another, to cover the infarct and border regions.
\end{abstract}

Results: The new regenerative cell delivery system using SMB sheets covered and wrapped with omentum resulted in (1) a significantly reduced infarct size causing, at least in part, a thin scar with thick well-vascularized cardiac tissue; (2) increased angiogenesis, as determined by a significantly higher vascular density; and (3) improved cardiac function, as determined by echocardiography, compared with the conventional method (SMB sheet implantation).

Conclusions: This cell delivery system shows potential for repairing the severely failed heart. ( $\mathrm{J}$ Thorac Cardiovasc Surg 2011;142:1188-96)

Heart failure is a frequent and life-threatening disorder, despite recent medical and surgical advances. Myocardial regenerative therapy is gaining interest as a means for improving left ventricular (LV) function in patients with end-stage heart disease. ${ }^{1-3}$ However, a recent clinical trial of cell transplantation by needle injection reported slightly disappointing results. ${ }^{2-4}$ The main drawbacks of cell transplantation by needle injection appear to be poor retention and survival of the injected cells, local mechanical myocardial damage owing to injury by the

From the Department of Cardiovascular Surgery, ${ }^{\text {a }}$ Osaka University Graduate School of Medicine, Suita, Osaka, Japan; and the Institute of Advanced Biomedical Engineering and Science, ${ }^{\mathrm{b}}$ Tokyo Women's Medical University, Tokyo, Japan.

Supported by Grants for the Research and Development of the Myocardial Regeneration Medicine Program from the New Energy Industrial Technology Development Organization (NEDO), Japan.

Disclosures: Authors have nothing to disclose with regard to commercial support.

Read at the 91st Annual Meeting of The American Association for Thoracic Surgery, Philadelphia, Pennsylvania, May 7-11, 2011.

Received for publication April 28, 2011; revisions received June 23, 2011; accepted for publication July 11, 2011; available ahead of print Sept 16, 2011.

Address for reprints: Yoshiki Sawa, MD, PhD, Department of Cardiovascular Surgery, Osaka University Graduate School of Medicine, Osaka, Japan (E-mail: sawa-p@surg1.med.osaka-u.ac.jp).

0022-5223/\$36.00

Copyright $₫ 2011$ Published by Elsevier Inc. on behalf of The American Association for Thoracic Surgery

doi:10.1016/j.jtcvs.2011.07.002 needle itself, and the potential for lethal arrhythmias. We have been investigating cell-sheet techniques for delivering cells to severely damaged myocardium more efficiently, without damaging the myocardium, and, consequently, more effectively. This technique provides better improvement of cardiac function than obtained with the needle cell-injection method. ${ }^{5-7}$

The greatest advantage of the cell-sheet technique is that the sheet consists only of cells, which produce an extracellular matrix without requiring an artificial scaffold. The cell sheet has a high ability to integrate with native tissues, because the adhesion molecules on its surface are preserved..$^{5-7}$ The layered grafts must be carefully prepared to avoid tearing, but they themselves are strong, flexible, and easy to work with.

It has been suggested that an increased number of implanted skeletal myoblast (SMB) sheets is related to better results, such as improved cardiac function and angiogenesis, less fibrosis, and less hypertrophy, with the amounts of secreted cytokines dependent on the number of cell sheets used. ${ }^{7}$ However, cell sheets with more than 5 layers show areas with disorganized vasculature, presumably because of insufficient supplies of blood, oxygen, and nutritients. ${ }^{7,8}$ Thus, in applying cell transplantation to the severely damaged myocardium, a key challenge is in improving the blood perfusion of the implanted cells so 

Abbreviations and Acronyms
LAD $=$ left anterior descending coronary
$\mathrm{LV}=$ left ventricular
LVEDV = left ventricular end-diastolic volume
LVEF = left ventricular ejection fraction
LVESV = left ventricular end-systolic volume
MI = myocardial infarction
$\mathrm{O}$ group $=$ omentum only
RT- $\quad=$ real-time polymerase chain reaction
PCR
$\mathrm{S}$ group $=$ cell sheets only
$\mathrm{SMB}=$ skeletal myoblast
$\mathrm{SO}=$ cell sheets wrapped with omentum
group
$\begin{aligned} \text { STAT3 }= & \text { signal transducer and activator of } \\ & \text { transcription } 3 \\ \text { VEGF } & \text { vascular endothelial growth factor }\end{aligned}$

that large numbers of regenerative cells can be delivered with minimal cell loss.

The omentum is reported to potentially provide revascularization for the ischemic myocardium, ${ }^{9}$ release a number of angiogenic cytokines, ${ }^{10,11}$ supply stem cells, and attenuate inflammation. ${ }^{12}$ The omentum was once commonly used in surgical revascularization to treat ischemic heart disease; however, omentopexy alone is not very effective for supporting the angiogenesis needed in the infarcted area for rapid recovery. ${ }^{9}$ On the basis of those findings, we speculated combining SMB sheets with omentum might enhance survival of the implanted cells by improving angiogenesis. Thus, as a novel method for implanting large amounts of cells, we developed a cell-delivery system using SMB sheets wrapped and covered with omentum flap as an external source for blood flow. We hypothesized that this method could replace the myocardial infarction (MI) scar with cell-sheet-based cardiac tissue in the pig heart.

\section{MATERIALS AND METHODS}

All studies were performed with the approval of the institutional ethics committee of Osaka University. Humane animal care was used in compliance with the "Principles of Laboratory Animal Care" formulated by the National Society for Medical Research and the "Guide for the Care and Use of Laboratory Animals" prepared by the Institute of Animal Resources and published by the National Institutes of Health (Publication No 85-23, revised 1996). The authors had full access to the data and take full responsibility for its integrity. All the authors have read and agreed to the manuscript as written. All procedures and evaluations, including the assessment of cardiac parameters, were carried out in a blinded manner.

\section{Animal Models and Study Protocol (Figure 1)}

Thirty-seven female mini-pigs (8-10 months old; Japan Farm Co Ltd, Kagoshima, Japan) weighing 20 to $25 \mathrm{~kg}$ were used in these experiments.

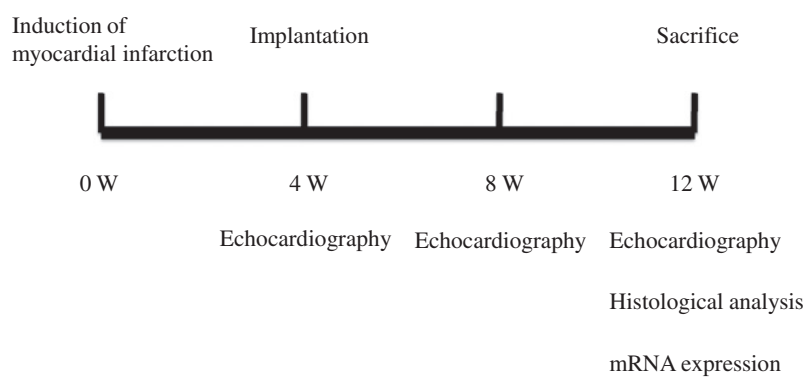

FIGURE 1. Study protocol for the assessment of cardiac function and histologic analysis.

The mini-pigs were anesthetized with an intravenous administration of ketamine $(6 \mathrm{mg} / \mathrm{kg})$ and sodium pentobarbital $(10 \mathrm{mg} / \mathrm{kg})$ for endotracheal intubation and then maintained with inhaled sevoflurane $(15 \%-2 \%)$. The pericardial space was exposed by left thoracotomy through the fourth intercostal space. The distal portion of the left anterior descending coronary artery (LAD) was directly ligated as ischemic preconditioning to reduce the occurrence of lethal ventricular arrhythmia, followed by placement of an ameroid constrictor around the LAD just distal to the left circumflex coronary artery branching. ${ }^{5,13}$ The muscle and skin were closed in layers, and the mini-pigs were then taken off the anesthetics. Eleven $(30 \%)$ of the study animals died in the early postoperative period. This technique produced an ischemic cardiomyopathy model that reflected clinical relevance and can be used for appropriate preclinical studies with minimal procedurerelated mortality.

Computer-generated random allocation generated 4 randomized study groups 1 week after MI. Autologous cells were isolated and grown in culture for 3 weeks to prepare samples for implantation. Four weeks after MI induction, the mini-pigs were again placed under general anesthesia for echocardiography followed by either cell-sheet implantation or a sham operation. Two mini-pigs whose LV ejection fractions (LVEFs) were above $40 \%$ before treatment, as measured by transthoracic echocardiography using the Simpson method, were excluded from the study. At 4 and 8 weeks after either cell-sheet implantation or sham operation, the mini-pigs again underwent general anesthesia for echocardiographic examination. The mini-pigs were humanely killed after the 8-week echocardiography measurements for histologic and biochemical analyses of the heart tissue.

\section{Preparation and Grafting of SMB Cell Sheets}

Autologous skeletal muscle weighing approximately 10 to $15 \mathrm{~g}$ was removed from the quadriceps femoris muscle, and purified autologous SMB cells were cultured for 3 weeks to prepare them for implantation, as described previsously. ${ }^{5}$ Autologous SMBs are precursor cells of adult myofibers and feature several advantages, including autologous origin, high in vitro scalability, lack of tumorigenicity (owing to their myogenic lineage restriction), and strong resistance to hypoxia after ischemia. The cells were incubated in 60-mm temperature-responsive culture dishes (UpCell; Cellseed, Tokyo, Japan) at $37^{\circ} \mathrm{C}$ for 24 hours $\left(1.5 \times 10^{7}\right.$ cells per dish). The dishes were then transferred to another incubator, set at $20^{\circ} \mathrm{C}$, for 1 hour to release the cultured cells as intact cell sheets. Under this protocol, the SMBs spontaneously detached from the plate as a free-floating monolayer cell sheet.

\section{Grafting the SMB Cell Sheet Wrapped With Omentum}

The mini-pigs with MI were divided into 4 treatment groups $(\mathrm{n}=6$ in each): cell sheets wrapped with omentum (SO group), cell sheets only (S group), omentum only (O group), and sham operation (sham group). Each animal in the SO and S groups received approximately 30 cell sheets $\left(1.5 \times 10^{7}\right.$ cells per sheet $)$ with the total cell number being $4.5 \times 10^{8}$. 
In the $\mathrm{S}$ group, a median sternotomy was performed. The cell sheets were placed on the epicardium of the ischemic area (LAD region) and stitched in place around the edge. In the SO group, a small upper midline laparotomy and median sternotomy were performed to move omentum from the peritoneal space into the mediastinal space, preserving the arch structure of the left gastroepiploic artery. We created a hole in the diaphragm and passed the omentum flap through the hole into the pericardial cavity. Five-layer cell sheets of SMBs were wrapped with omentum and then covered with the harvested omental flap, because a maximum of 5 cell-sheet layers can be implanted in one place. ${ }^{7,8}$ Six 5-layer constructs were each placed on a different area to cover the infarct and border regions. The operations were performed with the animals under general anesthesia.

\section{Histologic and Immunohistochemical Analyses}

Eight weeks after the treatment, the hearts were dissected and embedded in optimum cutting temperature compound, snap-frozen in liquid nitrogen, and cut into 5- $\mu \mathrm{m}$ thick sections. The paraffin-embedded sections were fixed in $4 \%$ paraformaldehyde and stained with hematoxylin-eosin (Figure 2) or Masson trichrome (Figure 3). The percentage of infarct area was quantified as the positively stained LV area/total LV area. The stained and total LV areas were obtained by tracing using Image $\mathbf{J}$ software at the mid-LV level, where the base of the papillary muscles is clearly depicted.

So that vascular density could be evaluated in the border area, the cryosectioned samples were immunolabeled with anti-von Willebrand factor antibody (1:250 dilution; Dako, Glostrup, Denmark) (Figure 4). The number of positively stained capillary vessels that were 5 to 10 $\mu \mathrm{m}$ in diameter in the peri-infarct border-zone myocardium in 16 individual randomly selected fields per heart was counted under highpower magnification $(\times 200)$. The numbers from the 16 fields were averaged to determine the vascular density (per square millimeter). The stained slides were viewed on a BioZero laser scanning microscope (Keyence, Osaka, Japan).

The following antibodies were used to identify SMBs: the primary antibodies were anti-smooth muscle actin, anti-vimentin, anti-desmin, and anti-skeletal myosin (fast) (all from Dako); the secondary antibodies were goat anti-mouse and anti-rabbit immunoglobulin G (Invitrogen, Leek, The Netherlands).

\section{Analysis of mRNA Expression}

Total RNA was extracted from cardiac muscle tissue, reverse transcribed into cDNA using TaqMan reverse transcription reagents (Applied Biosystems, Stockholm, Sweden), and real-time polymerase chain reaction (RT-PCR) was performed with an ABI PRISM 7700 machine (Applied Biosystems). ${ }^{14}$ For each gene, RNA samples were prepared and assayed in triplicate. RT-PCR was used to determine the expressions of vascular endothelial growth factor (VEGF) and signal transducer and activator of transcription 3 (STAT3) in our in vivo experiments. The average copy number of gene transcripts for each sample was normalized to that for glyceraldehyde-3-phosphate dehydrogenase.

\section{Echocardiography: Measurements of Global LV Function}

Global cardiac function was assessed using commercially available echocardiographic equipment with a $4.0-\mathrm{MHz}$ transducer (Aplio; Toshiba, Otawara, Japan) before and 4 and 8 weeks after cell-sheet implantation. Echocardiographic measurements included LV end-diastolic and end-systolic volumes (LVEDV and LVESV, respectively) and LVEF. LVEF was calculated as follows: $\operatorname{LVEF}(\%)=100 \times(\operatorname{LVEDV}-\mathrm{LVESV}) /(\mathrm{LVEDV})$.
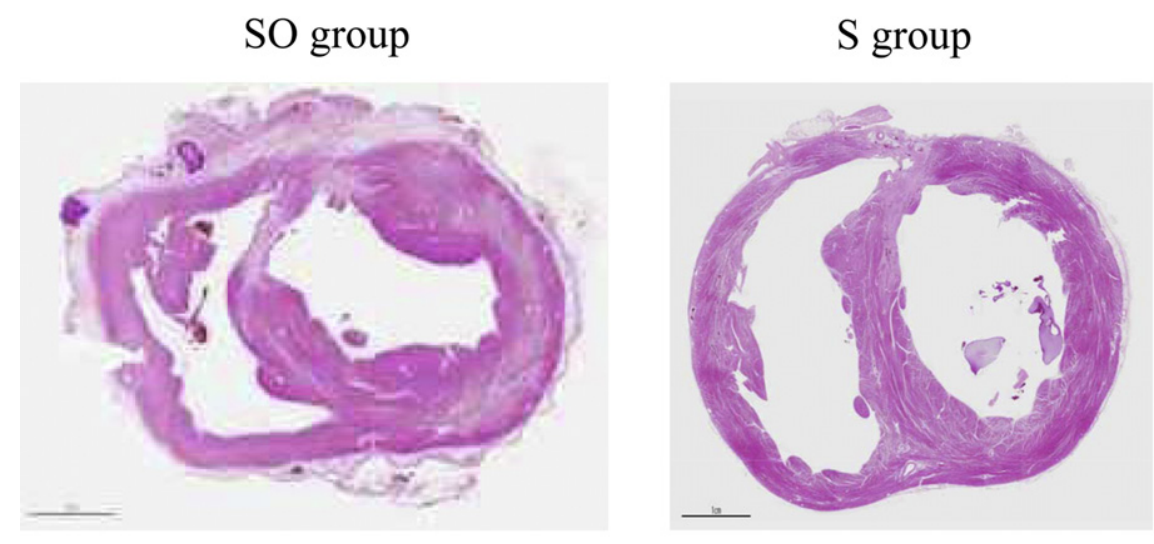

\section{O group}
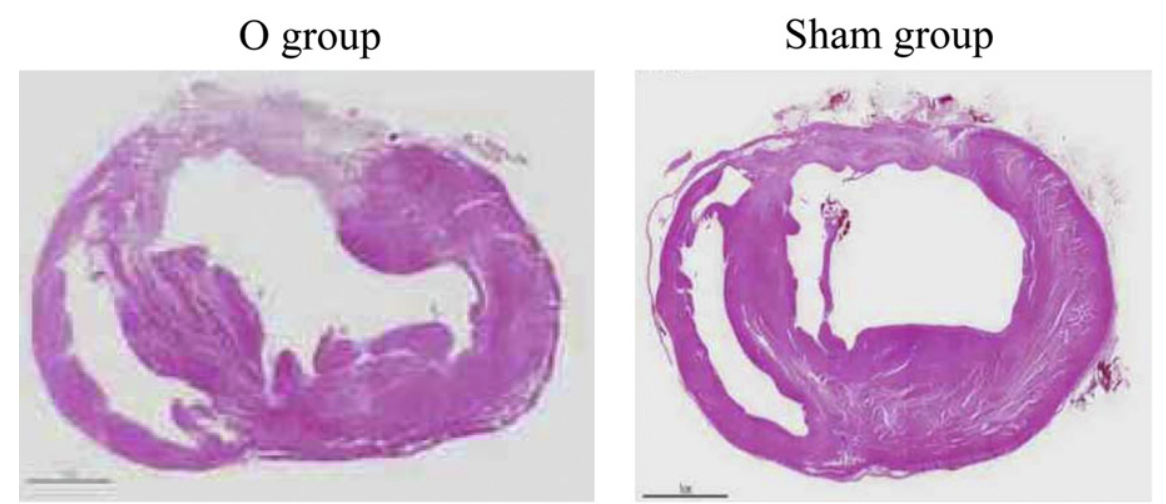

FIGURE 2. Macroscopic $(\times 40)$ views of the heart in the 4 groups (hematoxylin-eosin staining). SO group, skeletal myoblast (SMB) sheets wrapped with omentum; $S$ group, SMB sheets only; O group, omentum only; sham group, sham operation. 

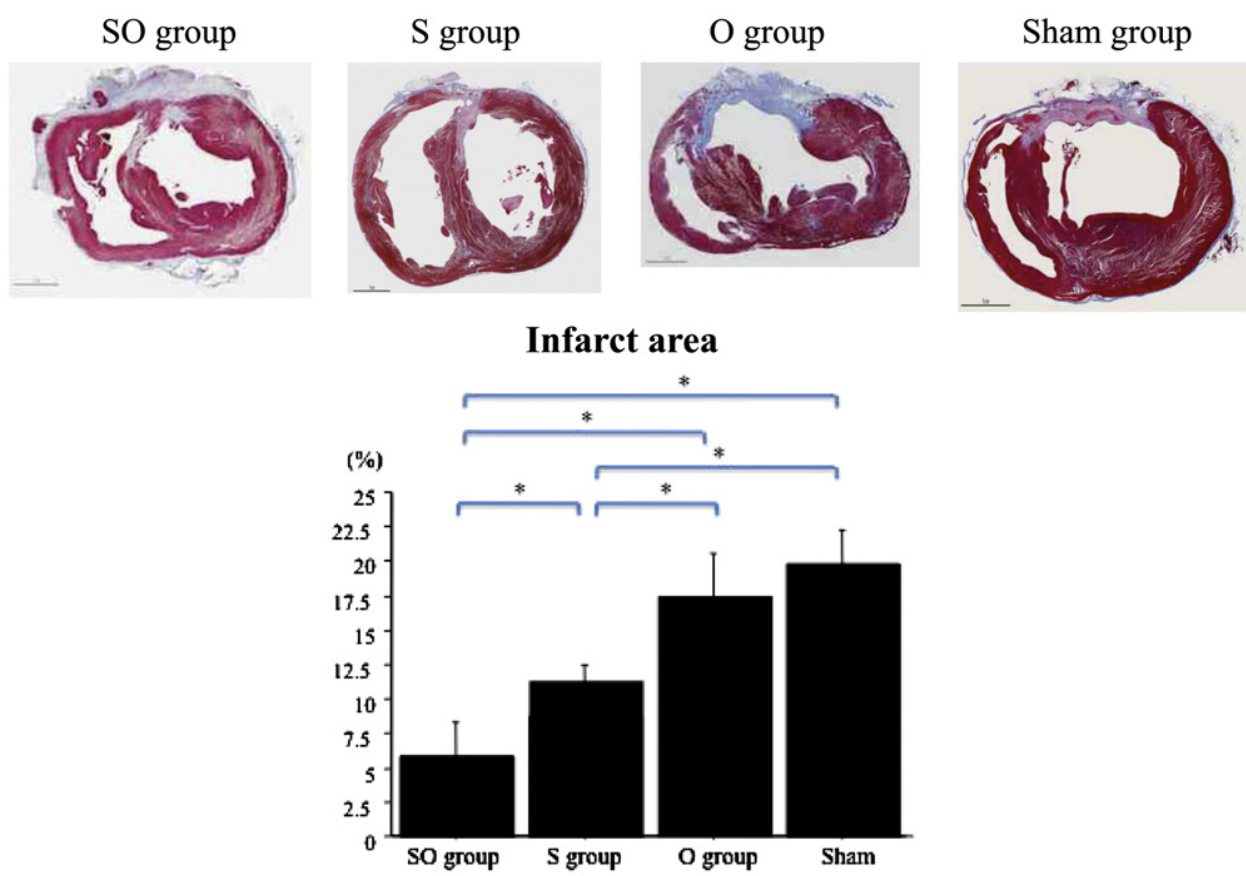

FIGURE 3. Macroscopic $(\times 40)$ views of the heart in the 4 groups (Masson trichrome staining). Infarct area: The SO group showed a significant improvement in the infarct area of the whole heart. Abbreviations as in Figure 2. $* P<.05$.

\section{Statistical Analysis}

SPSS software (version 11.0, SPSS, Inc, Chicago, Ill) was used for statistical analyses. Continuous values are expressed as the mean \pm standard deviation. The significance of differences was determined using a 2-tailed multiple $t$ test with Bonferroni correction following analysis of variance for individual differences.

\section{RESULTS}

\section{Modulation of Myocardial Structure}

Myocardial structural components, including fibrosis and vascularity, were assessed by hematoxylin-eosin staining, Masson trichrome staining, and immunohistochemistry
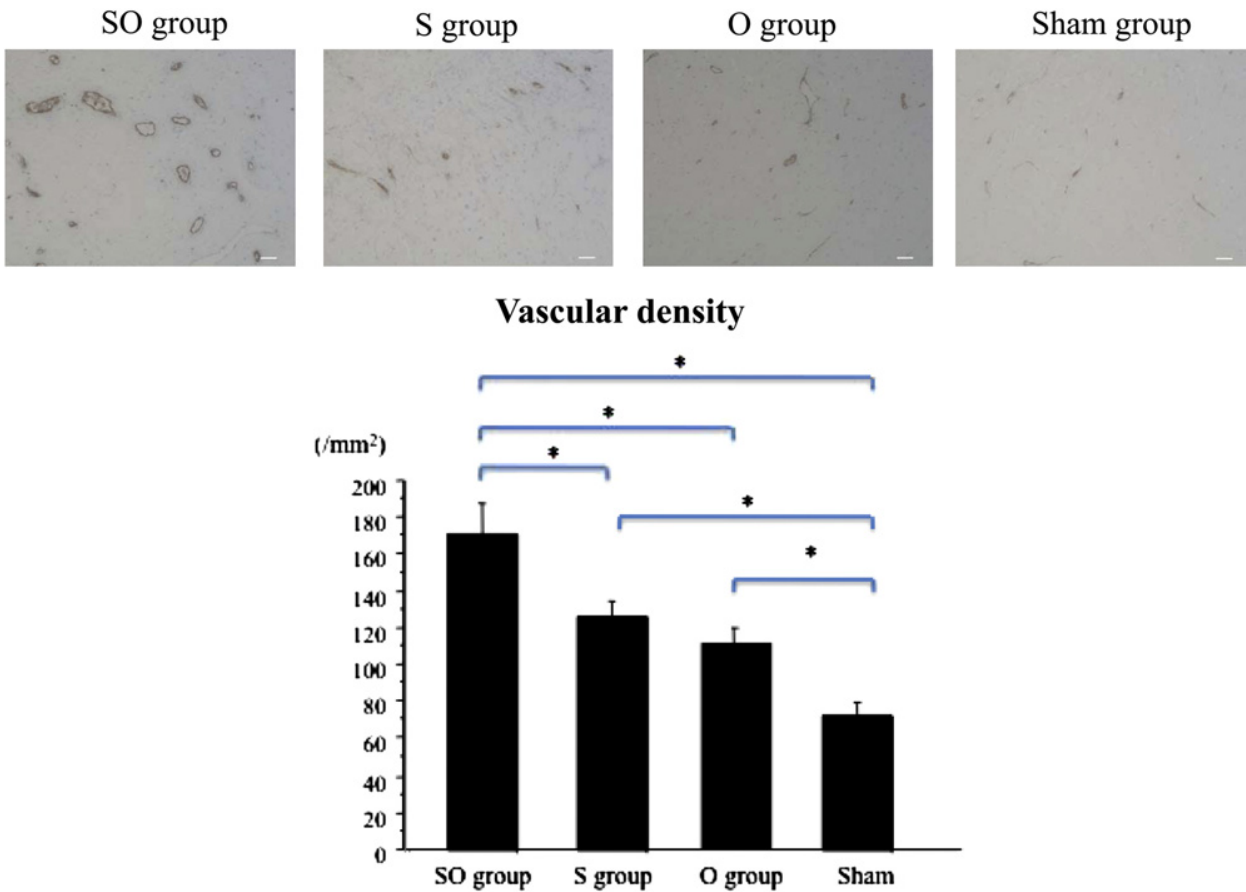

FIGURE 4. Microscopic $(\times 200)$ views of sections of the peri-infarct border-zone region stained with the von Willebrand factor antibody (factor VIII) in the 4 groups $($ bar $=20 \mu \mathrm{m})$. Vascular density: The SO group showed a significant improvement in vascular density, as assessed by anti-von Willebrand factor antibody. Abbreviations as Figure $2 . \mathrm{N}>4$ in each group. $* P<.05$. 

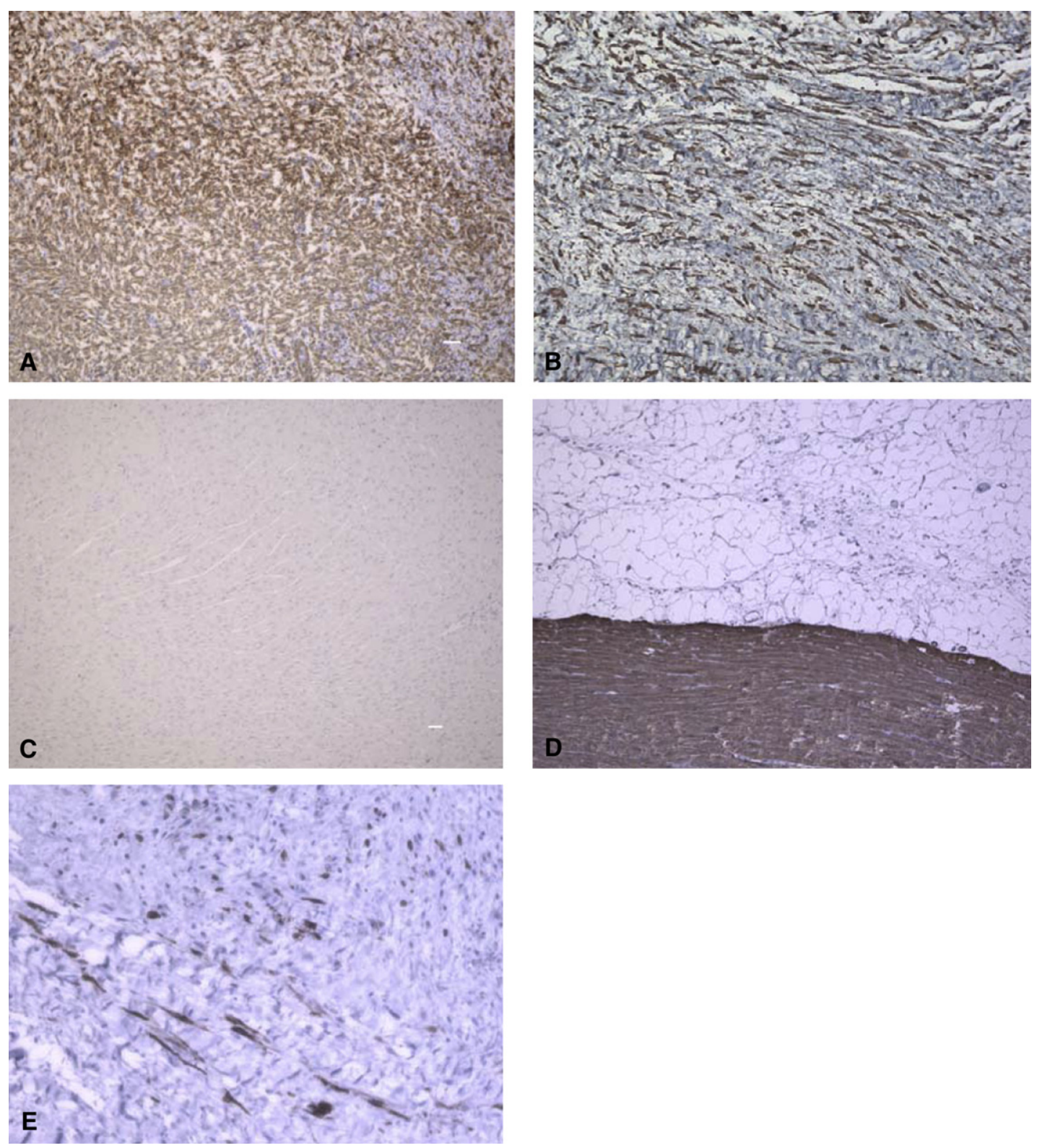

FIGURE 5. A, Microscopic $(\times 200)$ views of the infarct region in the SO group (alpha smooth muscle actin staining). B, Microscopic $(\times 200)$ views of the infarct region in the SO group (vimentin staining). C, Microscopic $(\times 200)$ views of the infarct region in the SO group (fast type myosin heavy chain staining). D and E, Microscopic $(\times 200)$ views of the heart (desmin staining). SO group, SMB sheets wrapped with omentum.

for anti-von Willebrand factor 8 weeks after treatment. The cavity of the LV was enlarged in the $\mathrm{O}$ and sham groups, whereas the global myocardial structure was well maintained in the SO and S groups, as assessed by hematoxylin-eosin staining (Figure 2). Collagen was densely accumulated in the infarct area and globally distributed in the remote area after the sham operation, whereas less collagen had accumulated in both the infarct and remote areas in the other 3 groups compared with the sham group, as assessed by Masson trichrome staining. The size of the infarct area, quantitatively assessed by computer-based planimetry of Masson trichrome-stained hearts, was significantly smaller in the SO group than in the other 3 groups (Figure 3). The vascular density, assessed by immunohistochemistry for anti-von Willebrand factor, was significantly greater in the SO group than in the other 3 groups (Figure 4).

Many alpha-smooth muscle actin- and vimentin-positive cells were present in the implanted sheets in the SO group; these cells were negative for fast-type myosin heavy chain and desmin (Figure 5, $A-D$ ). However, a few desminpositive cells were also detected at the implanted site (Figure 5, E). These results suggested that most of the 
detected cells had the phenotype of myofibroblasts, whereas a few expressed the phenotype of SMBs. Although these cells might have played an important role in the myocardial regeneration after SMB sheet implantation, their origin and characteristics have not been fully evaluated. Further study will be needed to elucidate the function of these cells and clarify the mechanisms of myocardial regeneration.

\section{mRNA Expressions After Cell-Sheet Implantation}

The expression of a variety of molecules that are intramyocardially expressed and potentially related to reverse LV remodeling was assessed by RT-PCR. Among them, the relative expression levels of VEGF and STAT3 mRNA were highest in the SO group, suggesting that the combination of SMB sheets and omentum accelerated the secretion of angiogenesis-related cytokines (ie, VEGF and STAT3) from the SMB sheets in vivo (Figure 6).

\section{Cardiac Functional Recovery}

Serial changes in the global systolic and diastolic LV function after cell-sheet implantation were assessed by conventional echocardiography. After the sham operation, LVEDV and LVESV tended to increase until 8 weeks, whereas the LVEF and anterior wall thickness did not change. In contrast, LVEDV did not change in the SO group, but LVESV tended to decrease in the SO and S groups. LVEF increased significantly at 4 and 8 weeks after treatment in the SO group compared with the baseline value. At 4 and 8 weeks after treatment, LVESV was significantly smaller and LVEF significantly greater in the SO group than in the $\mathrm{S}$ group, whereas there was no significant difference in LVEDV between them. The anterior wall

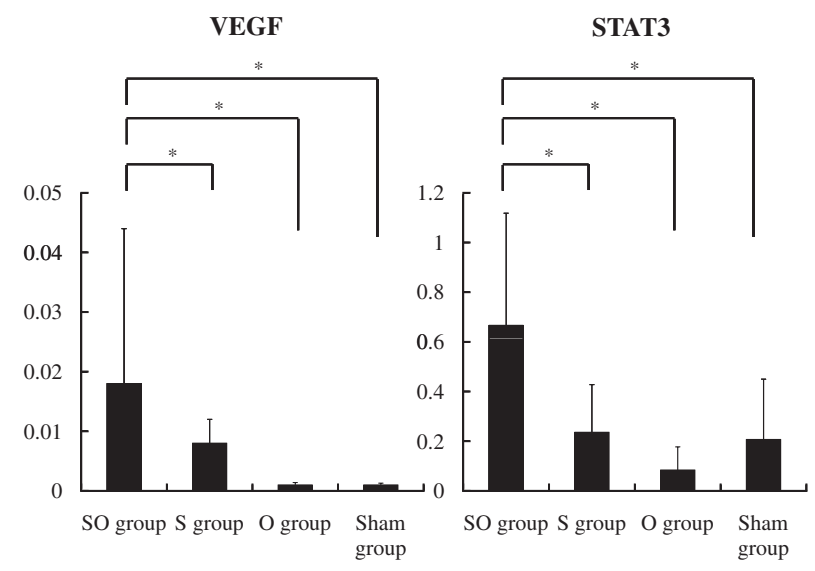

FIGURE 6. mRNA expressions in implanted infarcted hearts were determined by real-time PCR using porcine primers. The expressions of vascular endothelial growth factor (VEGF) and signal transducer and activator of transcription 3 (STAT3) mRNA were significantly increased in the SO group compared with the other groups. Abbreviations as in Figure 2. $\mathrm{N}>4$ in each group. $* P<.05$. thickness increased significantly at 8 weeks after treatment in the SO group compared with its baseline value; this change was significantly larger than that in the sham group (Figure 7).

\section{DISCUSSION}

The major findings of this study were that a new regenerative cell delivery system using SMB sheets covered and wrapped with omentum resulted in the following benefits compared with the conventional method (ie, SMB sheet implantation): (1) a reduced infarct area that led, at least in part, to a thin scar with thick well-vascularized cardiac tissue; (2) angiogenesis induction; and (3) improved cardiac function. Our data suggest that this new cell-delivery system acted in large part by enhancing the paracrine effect of the SMBs.

The mechanism of the restoration of damaged myocardium by SMB sheet implantation is complex, involving many pathways. ${ }^{5-7}$ Recent reports describe the beneficial results of SMB sheet implantation in several animal experimental models and patients with heart failure. These benefits were primarily attributed to the secretion of cytokines from the implanted cell sheets (ie, a paracrine effect). It has been suggested that regenerative therapy for the severely damaged myocardium, in which large numbers of cells must be delivered with minimal cell loss, may be achieved by improving the blood perfusion in the region of implanted cells. ${ }^{7,8}$ The general intention is to protect the implanted cells from ischemic injury and necrosis by inducing cardiac protective responses (eg, angiogenesis and attenuation of inflammation). A previous study ${ }^{15}$ reported that angiogenic factors, such as endothelial cells and some angiogenic growth factors, can enhance angiogenesis to improve the survival of thick-layered cardiomyocyte sheets applied to the damaged myocardium. Shimizu and associates ${ }^{8}$ reported that transplanted 3-layered cardiomyocyte sheets could be vascularized in subcutaneous tissue without necrosis, but sheets with 4 or 5 layers had areas with disorganized vasculature and primary ischemia. To overcome this limitation, they performed repeated transplantations of triple-layer grafts, which created a thick myocardium with a wellorganized microvascular network. ${ }^{8}$ However, technical hurdles still need to be overcome before a similar method can be clinically applied in humans.

In the present study, we demonstrated that the implantation of SMB sheets in combination with omentum was superior to conventional SMB-sheet implantation. The use of an omentum flap to provide revascularization within the ischemic myocardium was previously performed in patients with ischemic heart disease. ${ }^{9}$ Although omentopexy alone was not effective for eliciting a rapid recovery, recent basic research studies have suggested a mechanism by which omentum tissue induces angiogenesis. ${ }^{10,11}$ 
LVEDV

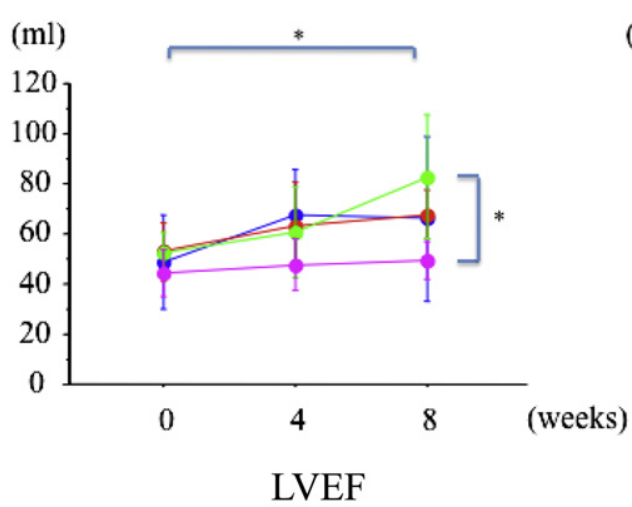

\section{LVESV}

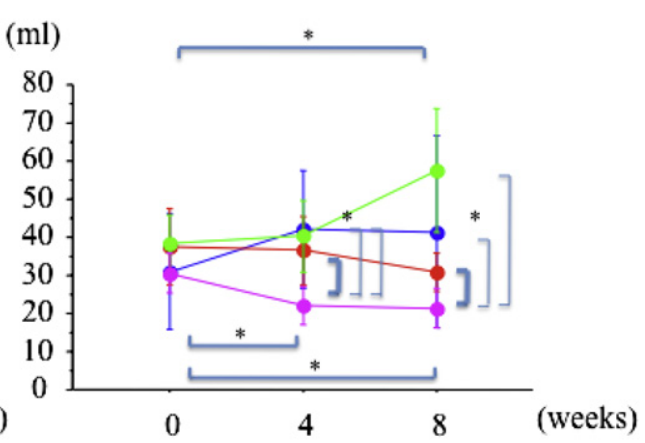

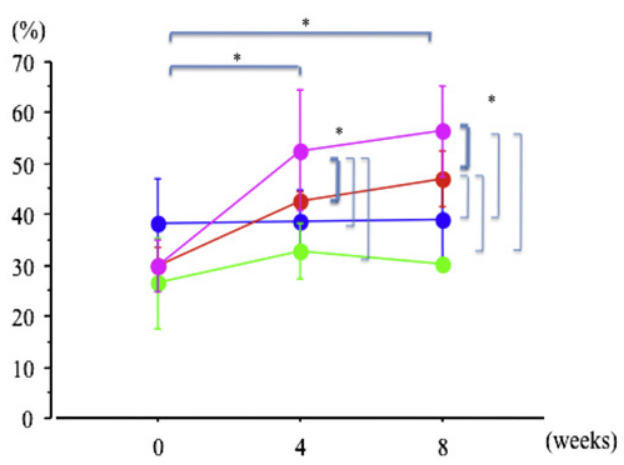

FIGURE 7. Echocardiographic analysis. The SO group showed less ventricular remodeling and greater degrees of improved anterior wall thickness and cardiac function than did the S group. Pink line, SO group; red line, S group; blue line, O group; green line, sham group; LVEDV, left ventricular end-diastolic volume; $L V E S V$, left ventricular end-systolic volume; $L V E F$, left ventricular ejection fraction; other abbreviations as in Figure 2 . $\mathrm{N}=6$ in each group. $* P<.05$.

Omentum flap has often been used by cardiothoracic surgeons to stimulate revascularization. Omentum also has the potential to supply fat-derived stem cells ${ }^{9}$ and various cytokines ${ }^{10,11}$ and to attenuate inflammation. ${ }^{12}$ The adipocytes in omentum release a number of angiogenic growth factors, such as VEGF, suggesting that omentum flap can act as a physiologic exogenous source of multiple angiogenic factors that act synergistically to promote arteriogenesis. ${ }^{16}$ Our results support the idea that the interposed omentum enhances the cardiac protection provided by the superimposed SMB sheets, such as by inducing angiogenesis, increasing the blood flow, and prolonging cell survival (in part by attenuating inflammation and protecting the cells from apoptosis). Other experimental studies aimed at enhancing the effects of omentopexy have been reported. Ruel and associates ${ }^{16}$ demonstrated that a gastric submucosal patch has excellent angiogenic effects when used as an endogenous source of growth factors in a swine model of chronic myocardial ischemia. Kanamori and colleagues ${ }^{17}$ showed that omentopexy enhances the angiogenic effect of cell therapy in a swine model of acute MI. Compared with these studies, our new method seems safer and less invasive because it does not require gastrectomy or bone marrow aspiration.
On the basis of our present results, we speculate that the myocardial functional recovery obtained with the combined method was associated with the upregulation of angiogenic cytokines (eg, VEGF, STAT3) and with increased angiogenesis and blood flow. In addition, the reduced infarct area, as determined by evaluating Masson trichrome-stained hearts, indicates that the animal model used in the present study may be one of hibernating myocardium rather than of chronic infarction. ${ }^{13}$ Together with the paracrine effects of the implanted SMB sheet, humoral substances from the omentum might have had beneficial effects on the native cardiomyocytes and viable surrounding muscle cells, preventing global myocardial remodeling. One possible mechanism to explain our results is that the SMB sheet with omentum implantation therapy induced the release of cytokines and enhanced the development of the microvasculature (ie, microcirculation, which is particularly vulnerable to injury during ischemia), which on reperfusion rescued the hibernating myocardium, thereby enhancing the recovery of myocardial performance. A recent report mentioned a possible role of stromal cell-derived factor 1 in recruiting stem cells. Because stromal cell-derived factor 1 is secreted in muscle tissue, and muscle satellite cells express functional CXCR4 receptors, the implanted SMBs may serve 
to recruit stem cells to the site of damage, where they promote heart repair. ${ }^{6}$ Nevertheless, it remains to be determined what cytokines play a major role in generating therapeutic effects among the many complex molecular and cellular mechanisms involved.

We could find no ventricular premature beat by Holter electrocardiographic analysis after the treatment (data not shown). $\mathrm{We}^{5}$ previously reported that rat and porcine MI models show less arrhythmia after being treated with SMB-sheet implantation than with needle injection. We speculate that the needle damages the myocardium, which may induce arrhythmia. In contrast, the SMB sheet implantation technique does not normally injure the myocardium. Moreover, we did not observe any cases of lethal ventricular arrhythmia in animals implanted with SMB sheets wrapped with omentum.

\section{CONCLUSIONS}

A new cell-delivery method using SMB sheets combined with omentum allowed us to implant large numbers of SMBs in a porcine MI model. The implanted sheets became well-vascularized cardiac tissue, with less scarring and improved cardiac function over that of the animals receiving SMB sheets alone. This method may be applicable for repairing the severely failing heart.

We thank Professor Nariaki Matsuura and Dr Naomasa Kawaguchi of the Department of Pathology, Osaka University Graduate School of Medicine, and Mr Akima Harada for the histologic analysis, Mr Shigeru Matsumi for the excellent technical assistance, Mrs Masako Yokoyama for RT-PCR, and Mrs Yuka Fujiwara and Mr Hiroyuki Shimada for cell culturing. We also thank Dr Leslie Miglietta and Dr Grace Gray for editing our manuscript.

\section{References}

1. Menasché P, Hagege AA, Scorsin M, Puzet B, Desnos B, Schwartz K, et al. Myoblast transplantation in heart failure. Lancet. 2001;357:279-80.

2. Ghostine S, Carrion C, Souza LC, Richard P, Bruneval P, Vilquin JT, et al. Longterm efficacy of myoblast transplantation on regional structure and function after myocardial infarction. Circulation. 2002;106(12 Suppl. 1):I131-6.

3. Hagege AA, Marolleau JP, Vilqiun JT, Alheritiere A, Peyrard S, Duboc D, et al. Skeletal myoblast transplantation in ischemic heart failure: long-term follow-up of the first phase I cohort of patients. Circulation. 2006;114(1 Suppl):I108-13.

4. Menasché P, Hagege AA, Vilquin JT, Desnos M, Abergel E, Pouzet B, et al. Autologous skeletal myoblast transplantation for severe postinfarction left ventricular dysfunction. J Am Coll Cardiol. 2003;41:1078-83.

5. Miyagawa S, Sawa Y, Sakakida S, Taketani S, Kondoh H, Memon IA, et al. Tissue cardiomyoplasty using bioengineered contractile cardiomyocyte sheets to repair damaged myocardium: their integration with recipient myocardium. Transplantation. 2005;80:1586-95.

6. Memon IA, Sawa Y, Fukushima N, Matsumiya G, Miyagawa S, Taketani S, et al. Repair of impaired myocardium by means of implantation of engineered autologous myoblast sheets. J Thorac Cardiovasc Surg. 2009;130:646-53.

7. Sekiya N, Matsumiya G, Miyagawa S, Saito A, Shimizu T, Okano T, et al. Layered implantation of myoblast sheets attenuates adverse cardiac remodeling of the infarcted heart. J Thorac Cardiovasc Surg. 2009;138:985-93.

8. Shimizu T, Sekine H, Yang J, Isoi Y, Yamato M, Kikuchi A, et al. Polysurgery of cell sheet grafts overcomes diffusion limits to produce thick, vascularized myocardial tissues. FASEB J. 2006;20:708-10.

9. O'Shaugnessy L. Surgical treatment of cardiac ischemia. Lancet. 1937;232: 185-94.
10. Tabata K, Jiang C, Nemoto S, Saji Y, Ikeda T, Urayama S, et al. A combination of omental flap and growth factor therapy induces arteriogenesis and increased myocardial perfusion in chronic myocardial ischemia: evolving concept of biologic coronary artery bypass grafting. J Thorac Cardiovasc Surg. 2006;132:891-9.

11. Shrager JB, Wain JC, Wright CD, Donahue DM, Vlahakes GJ, Moncure AC, et al. Omentum is highly effective in the management of complex cardiothoracic surgical problems. J Thorac Cardiovasc Surg. 2003;125:526-32.

12. Levashev YN, Akopov AL, Mosin IV. The possibilities of greater omentum usage in thoracic surgery. Eur J Cardiothorac Surg. 1999;15:465-8.

13. Teramoto N, Koshino K, Yokoyama I, Miyagawa S, Ose T, Zeniya T, et al. Experimental pig model of old myocardial infarction with long survival leading to chronic LV dysfunction and remodeling as evaluated by PET. J Nucl Med 2011;52:761-8. Epub 2011 Apr 15.

14. Horiguchi K, Sakakida-Kitagawa S, Sawa Y, Li ZZ, Fukushima N, Shirakura R, et al. Selective chemokine and receptor gene expressions in allografts that develop transplant vasculopathy. J Heart Lung Transplant. 2002;21:1090-100.

15. Sekine H, Shimizu T, Hobo K, Sekiya S, Yang J, Yamato M, et al. Endothelial cell coculture within tissue-engineered cardiomyocyte sheets enhances neovascularization and improves cardiac function of ischemic hearts. Circulation. 2008; 118(Suppl I):S145-52.

16. Ruel MA, Sellke FW, Bianchi C, Khan TA, Faro R, Zhang JP, et al. Endogenous myocardial angiogenesis and revascularization using a gastric submucosal patch. Ann Thorac Surg. 2003;75:1443-9.

17. Kanamori T, Watanabe G, Yasuda T, Nagamine H, Kamiya H, Koshida Y. Hybrid surgical angiogenesis: omentopexy can enhance myocardial angiogenesis induced by cell therapy. Ann Thorac Surg. 2006;81:160-8.

\section{Discussion}

Dr Vivek Rao (Toronto, Ontario, Canada). I have no relevant financial disclosures.

I thank the authors for the courtesy of providing me with a copy of their manuscript well in advance of the meeting. I congratulate them as well for a very elegantly performed and executed study, and I will confirm with the audience that the results shown here are just a small tip of the iceberg of the complete results presented in the manuscript. Most important, the manuscript contains a lot of functional data that, unfortunately, owing to time constraints, Dr Shudo was unable to present.

Having said that, I have a few questions with regard to this study. In the manuscript, it was not quite clear to me whether the number of cell sheets delivered were different between the omentum group (O group) and the cell-sheets-only group ( $\mathrm{S}$ group). You mentioned that to achieve the omentum wrapping you had a limitation of 5 sheets in the omentum-plus-cell group (SO group). Was that different from the total number of cell sheets implanted in the cell-sheets-only group (S group)?

Dr Shudo. Thank you for your useful comments, Dr Rao.

In this study we used 30 cell sheets total. On the basis of our results in the previous studies, the increase in the number of implanted cell sheets was related to the favorable results, such as enhancing the paracrine effect and improving cardiac function. However, these previous studies also suggested that more than 5 layers of SMB sheets had disorganized vasculature in the implanted site, maybe owing to the primary ischemia. Therefore, in this study, at most 5 layers were attached in one place and covered and wrapped with the omentum to support the angiogenesis.

Dr Rao. I will point to some of the LV function data that were presented in your manuscript. You measured LV function both at 4 weeks and 8 weeks after LAD ligation. What was striking to me was that in most models of LAD ligation there is continued deterioration in $\mathrm{LV}$ function in the control groups. In contrast, in your study, the 3 relative control groups had stabilization of their LV 
function from 4 to 8 weeks with a continued improvement in LV function in the omentum-plus-cell-transplant group (SO group). Can you explain why the LV function appeared to stabilize in your model.

Dr Shudo. In this study, MI was induced by first ligating the distal portion of the LAD for ischemic preconditioning, followed by placement of the ameroid constrictor around the main trunk of the LAD, just distal to the left circumflex artery branching. It has been reported that the ameroid ring was estimated to occlude inside completely about 2 weeks after being placed in vivo. As you mentioned (and as shown in one slide), LVEF seems to be unchanged 1, 2 , and 3 months after placement of the ameroid ring, whereas LVEDV and LVESV indices significantly increased gradually after induction of MI. Therefore, I would like to mention that LV remodeling was successfully obtained in this model in this study.

Dr Rao. I have 1 final question. You showed that the cell retention was approximately double that with the addition of the omentum wrapping, $60 \%$ cell retention versus $30 \%$ with the cell sheet alone. Yet for almost all of your end points there was absolutely no benefit to cell sheet implantation alone, which is obviously in contrast to many of the studies done previously with SMBs showing a benefit in function, angiogenesis, et cetera. Can you explain why your cell sheet transplant alone had absolutely no benefit in this model.
Dr Shudo. That seems to be a bit confusing. We have obtained good results even in the cell-sheet-only group (S group), too. However, we would like to focus on the preferable results of the new cell-implantation method.

Thank you again for your comments and good questions.

Dr Paul Kurlansky (Miami, Fla). I have no disclosures.

This was very interesting work. I have just 2 questions. First, what exact cells or mixture of cells are you putting into the cell sheets? Second, have you looked histologically at the hearts to see if you actually have myocardial regeneration or if you just have hypertrophy and preservation of the residual border zone myocardium?

Dr Shudo. Thank you so much. Regarding the first question, although this is unpublished information, we performed the experimental study about SMBs mixed with adipose tissue, derived mesenchymal stem cells. This model shows priority to the SMB cell-sheet-only group ( $\mathrm{S}$ group).

Regarding the analysis of border zone, our study using tissue Doppler echocardiography and speckle tracking echocardiography data showed that regional functional recovery was significantly greater in the infarct border region. This result may support the idea that cell-sheet-with-omentum therapy prevented progression of MI by supporting the hibernating myocardium. 